\title{
Electromagnetic properties of hadrons with two flavors of dynamical domain wall fermions*
}

\author{
Norikazu Yamada ${ }^{\dagger}$ \\ High Energy Accelerator Research Organization(KEK), Tsukuba, Ibaraki 305-0801, Japan \\ The Graduate University for Advanced Studies, Tsukuba, Ibaraki 305-0801, Japan \\ E-mail: norikazu.yamada@kek.jp \\ Thomas Blum \\ Physics Department, University of Connecticut, Storrs, CT 06269-3046, USA \\ RIKEN BNL Research Center, Brookhaven National Laboratory, Upton, New York 11973, USA \\ E-mail: tblum@phys.uconn.edu

\section{Masashi Hayakawa} \\ Theoretical Physics Group, RIKEN, Wako 2-1, Saitama 351-0198, Japan \\ E-mail: haya@postman.riken.go.jp

\section{Taku Izubuchi} \\ Institute for Theoretical Physics, Kanazawa University, Kanazawa 920-1192, Japan \\ RIKEN BNL Research Center, Brookhaven National Laboratory, Upton, New York 11973, USA \\ E-mail: izubuchi@quark.phy.bnl.gov

\section{for the RBC Collaboration}

We present the determination of the light quark masses using electromagnetic splittings of pions and kaons. The meson masses are calculated on $S U(3) \times U(1)$ gauge configurations, where $S U(3)$ gauge fields include sea quark effects of two degenerate flavors and the $U(1)$ gauge fields are incorporated in non-compact form. Possible sources of systematic uncertainty are discussed.

XXIIIrd International Symposium on Lattice Field Theory

25-30 July 2005

Trinity College, Dublin, Ireland

${ }^{*}$ We thank RIKEN, Brookhaven National Laboratory and the U.S. Department of Energy for providing the facilities and hospitality where this work was done.

†peaker. 


\section{Introduction}

Electromagnetic (EM) properties of hadrons offer a rich source of interesting and important phenomena. The patterns of the mass splittings between charged and neutral mesons or the mass splittings among the octet or decuplet baryons are sensitive to the isospin breaking of up and down quark masses and the presence of EM interactions. It is also known that the width difference of the $\rho^{+}$and $\rho^{0}$ mesons and the hadronic light-by-light scattering amplitude, which may not be computed from measured EM properties of hadrons, play an important role in the standard model (SM) prediction of the anomalous magnetic moment of muon.

Recent developments in lattice QCD in both hardware and software have advanced the fi eld closer to the goal of QCD calculations without approximation; large-scale, high precision unquenched simulations are becoming available [1]. What is important here is that the statistical error of pseudoscalar meson masses are well under control, with size typically a few percent or less. Remembering that charged-neutral meson splittings are of $O\left(\alpha_{\mathrm{em}}\right) \sim O(1 \%)$, where $\alpha_{\mathrm{em}}$ is the fi ne structure constant, it is expected that once EM interactions are successfully included, it will be possible to determine the up and down quark masses, which are the poorly known parameters of the SM, from fi rst principles by using such splittings as inputs, and thus we can check the simplest solution to the strong CP problem, $m_{u}=0$.

In this talk, we focus on the determination of the light quark masses. The strategy is basically following Refs. [2], where the EM fi elds are introduced in a non-compact form, and combined with QCD link variables to realize QCD + QED theory on the lattice. While this pioneering work made use of the Wilson quark action in the quenched approximation to QCD, here we employ domain wall fermions [3, 4] and QCD confi gurations with two flavors of dynamical domain wall fermions, generated by the RBC Collaboration [5]. Our activity toward the lattice calculation of the anomalous magnetic moment of muon is reported in Ref. [6].

\section{QCD+QED system}

We employ unquenched QCD gauge confi gurations $U_{\mathrm{qcd}, \mu}(x)$, generated with parameters $V=$ $16^{3} \times 32, L_{s}=12, M_{5}=1.8$ and three different sea quark masses, $m_{\text {sea }}=0.02,0.03,0.04$ [5]. The lattice spacing is determined to be $1.691(53) \mathrm{GeV}$ from $m_{\rho}=770 \mathrm{MeV}$, and hence the physical volume is roughly $(1.9 \mathrm{fm})^{3}$. Calculations are performed on about 200 confi gurations from the 5,000 trajectories available at each value of the sea quark mass.

A non-compact form is adopted for the action of the $U(1)$ gauge fi elds, $A_{\mathrm{em}, \mu}(x)$ [2]. To generate $A_{\mathrm{em}, \mu}(x)$, we first rewrite the action in momentum space, and impose the Coulomb gauge condition. After diagonalizing the kernel, the gauge fi elds are chosen randomly according to a Gaussian distribution and with $e=1$. The confi guration $A_{\mathrm{em}, \mu}(x)$ is then obtained by Fourier transformation. It is worth noting that with the use of the non-compact action and the generation procedure described above there is no auto-correlation among the confi gurations, even for arbitrarily small coupling. Since quenched QED is a free theory, the fi ne structure constant $\alpha_{\mathrm{em}}$ receives no renormalization. Using $A_{\mathrm{em}, \mu}(x)$ and the quark electric charge $Q_{q}$, we obtain a $U(1)$ link variable, $\left(U_{\mathrm{em} \mu}(x)\right)^{Q_{q}}=e^{i Q_{q} A_{\mathrm{em}, \mu}(x)}$. Confi gurations for the (QCD + QED) theory are then constructed by $U_{\mathrm{qcd}, \mu}(x) \times\left(U_{\mathrm{em}, \mu}(x)\right)^{Q_{q}}$, which are used in the inversion of the Dirac operator for valence quarks. 
Though $e=1$ is adopted in the confi guration generation, any value of the quark's electric charge can be realized by tuning $Q_{q}$.

In this system, the flavor non-singlet axial Ward-Takahashi (WT) identity for two flavors of domain wall fermions with mass $m_{q 1}$ and $m_{q 2}$ and charge $Q_{q 1}$ and $Q_{q 2}$ is given by

$$
\partial_{\mu}^{*} \mathscr{A}_{\mu}^{a}(x)=\left(m_{q 1}+m_{q 2}\right) P^{a}(x)+\left(m_{q 1}-m_{q 2}\right) \bar{q}(x) \gamma_{5}\left\{\frac{\tau^{a}}{2}, \frac{\tau^{3}}{2}\right\} q(x)+2 J_{5 q}^{a}(x)+\sum_{s} \varepsilon(s) \mathscr{X}_{s}^{a}(x),
$$

where $\mathscr{A}_{\mu}^{a}(x)$ takes the same form as the conserved axial-vector current in the pure QCD case, but with link variables, $U_{\mathrm{qcd}, \mu}(x) \times \operatorname{diag} .\left[\left(U_{\mathrm{em}, \mu}(x)\right)^{Q_{q 1}},\left(U_{\mathrm{em}, \mu}(x)\right)^{Q_{q^{2}}}\right], P^{a}(x)$ a pseudoscalar density, $J_{5 q}^{a}(x)$ the same as in pure QCD, and

$$
\begin{aligned}
& \mathscr{X}_{s}^{a}(x)=-\frac{1}{2} \sum_{\mu}\left[\bar{\Psi}_{s}(x)\left(1-\gamma_{\mu}\right) U_{\mathrm{qcd}, x, \mu}\left(\left(U_{\mathrm{em}, \mu}(x)\right)^{Q_{q 1}}-\left(U_{\mathrm{em}, \mu}(x)\right)^{Q_{q 2}}\right)\left[\frac{\tau^{a}}{2}, \frac{\tau^{3}}{2}\right] \Psi_{s}(x+\hat{\mu})\right. \\
& \left.+\bar{\Psi}_{s}(x)\left(1+\gamma_{\mu}\right) U_{\mathrm{qcd}, \mu}^{\dagger}(x-\hat{\mu})\left(\left(U_{\mathrm{em}, \mu}^{\dagger}(x-\hat{\mu})\right)^{Q_{q 1}}-\left(U_{\mathrm{em}, \mu}^{\dagger}(x-\hat{\mu})\right)^{Q_{q 2}}\right)\left[\frac{\tau^{a}}{2}, \frac{\tau^{3}}{2}\right] \Psi_{s}(x-\hat{\mu})\right],
\end{aligned}
$$

and $\varepsilon(s)=1$ for $1 \leq s \leq L_{s} / 2$ and -1 for $L_{s} / 2<s \leq L_{s}$. Notice that $\mathscr{X}_{s}^{a}(x)$ vanishes when $a=3$ or $Q_{q 1}=Q_{q 2}$.

From the analogy to the fact that in pure QCD with the domain-wall formalism the presence of the singlet axial anomaly becomes clear by considering the disconnected diagram involving the singlet, mid-point current, it is inferred that the similar understanding is possible for the $U(1)$ anomaly, but this time the disconnected diagram involving the flavor non-singlet, mid-point current $J_{5 q}^{a}(x)$ plays an important role. Due to the presence of the $U(1)$ anomaly, none of the components of $\mathscr{A}_{\mu}^{a}(x)$ is conserved for $Q_{q 1} \neq Q_{q 2}$ even in the quark massless limit, and hence there is no NambuGoldstone (NG) boson in this system [7]. If you consider the lattice measurement of $m_{\pi^{0}}$ through the standard method using two-point correlation functions, the possible origin of the non-vanishing $m_{\pi^{0}}$ is the $\pi^{0}$-singlet mixing, and it could affect $m_{\pi^{0}}$ by $O\left(\alpha_{\mathrm{em}}^{2}\right)$ or higher. At present, we do not include the $\pi^{0}$-singlet mixing. Thus, $\pi^{0}$ must become massless in the quark massless limit. Notice that even after including the mixing effects, it is justifi ed to regard $\pi^{0}$ as an NG boson up to $O\left(\alpha_{\mathrm{em}}^{2}\right)$. In addition to the above approximation, we are neglecting the disconnected diagram in $\pi^{0}-\pi^{0}$ correlation function (for now). It should be noted that the latter approximation does not affect the fact that " $\pi^{0}$ " becomes massless in the quark massless limit, and more importantly, the point where $m_{\pi^{0}}$ vanishes.

\section{Numerical results}

At present, we only have meson data in which the two valence quarks are degenerate with the sea quark mass. The electric charge of the valence quarks is set to $Q_{q}=+2 e / 3$ for the up quark and $-e / 3$ for the down quark. In this work, we take three values of $e$, which correspond to $\alpha_{\mathrm{em}}=\alpha_{\mathrm{em} \text {,phys }}, \frac{(0.6)^{2}}{4 \pi}, \frac{(1.0)^{2}}{4 \pi}$, to examine the $\alpha_{\mathrm{em}}$ dependence of the meson mass splittings. The mass of the pions is extracted from two-point correlation functions as usual. We employ two different operators, $P^{a}(x)$ and $A_{0}^{a}(x)$, as the interpolating operator. Since the EM interactions violate 


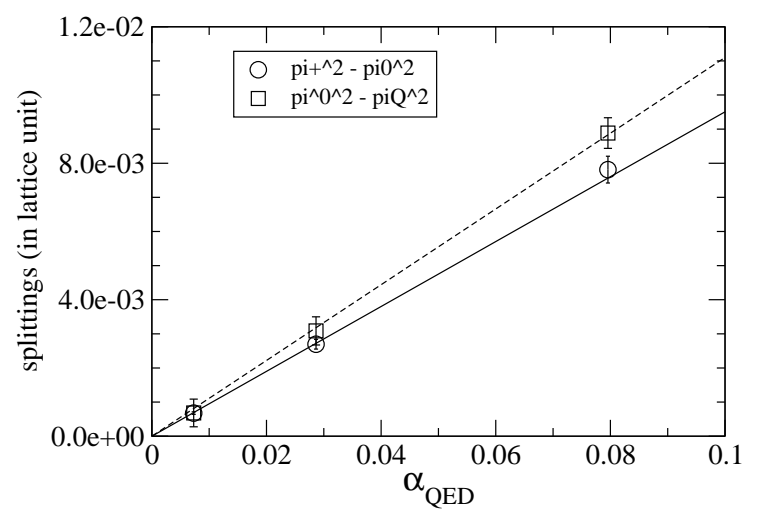

Figure 1: The $\alpha_{\mathrm{em}}$ dependence of the splittings. The results from $\left\langle A_{0} A_{0}\right\rangle$ are shown.

isospin symmetry, $\pi^{0}$ mixes with the lightest isosinglet state. However, this mixing turns out to start only at $O\left(\alpha_{\mathrm{em}}^{2}\right)$, and hence we neglect this effect as the present statistics cannot resolve such a small effect. Although the calculation of $m_{\pi^{0}}$ requires a disconnected diagram, we do not include it, as mentioned earlier. We will come back to this point later.

At the leading order of chiral perturbation theory (ChPT) including the EM interactions, $m_{\pi^{+}}^{2}$ and $m_{\pi^{0}}^{2}$ are given by

$$
\begin{array}{ll}
m_{\pi^{+}}^{2}=\alpha_{\mathrm{em}} \Delta_{+}^{(0)}+ & 2\left(B_{0}+\alpha_{\mathrm{em}} \Delta_{+}^{(m)}\right)\left(m_{f,+}+m_{\mathrm{res}}\right), \\
m_{\pi^{0}}^{2}= & 2\left(B_{0}+\alpha_{\mathrm{em}} \Delta_{0}^{(m)}\right)\left(m_{f,+}+m_{\mathrm{res}}\right),
\end{array}
$$

to $O\left(\alpha_{\mathrm{em}}^{2}\right)$, where $m_{f, \pm}=\left(m_{f, d} \pm m_{f, u}\right) / 2$. In the following, these functional forms are used to determine the low energy constants (LEC's), $B_{0}, \Delta_{+}^{(0)}, \Delta_{+}^{(m)}$ and $\Delta_{0}^{(m)} . m_{\text {res }}$ is estimated by finding $m_{f}$ at which $m_{\pi^{0}}^{2}$ vanishes, since $\pi^{0}$ can be considered as an NG boson.

Before discussing the determination of the LEC's, let us mention $O\left(\alpha_{\mathrm{em}}\right)^{2}$ corrections. Figure 11 shows the $\alpha_{\mathrm{em}}$ dependence of the $\pi^{+}-\pi^{0}$ and $\pi^{0}-\pi^{Q}$ mass splittings, where $\pi^{Q}$ denotes the pion in pure QCD. It is seen that each splitting is well described by a linear function of $\alpha_{\mathrm{em}}$ in the range $\frac{1}{137}<\alpha_{\mathrm{em}}<0.08$. This means that $O\left(\alpha_{\mathrm{em}}^{2}\right)$ contributions are not signifi cant in this range of $\alpha_{\mathrm{em}}$. This observation allows us to treat the EM interactions perturbatively.

Now we proceed to the determination of the LEC's. The entire analysis is done under a jackknife procedure. $B_{0}$ is obtained by fitting the pure-QCD pion data, $m_{\pi^{2}}^{2}$. By fitting $m_{\pi^{+}}^{2}-m_{\pi^{0}}^{2}$ as a function of $m_{f}$, the combination $\left(\Delta_{+}^{(m)}+\Delta_{0}^{(m)}\right)$ and $\Delta_{+}^{(0)}$ are extracted. Finally $\Delta_{0}^{(m)}$ is obtained from the slope of $m_{\pi^{0}}^{2}-m_{\pi^{Q}}^{2}$. In Fig. 2, the $m_{f}$ dependence of $m_{\pi^{+}}^{2}-m_{\pi^{0}}^{2}$ and $m_{\pi^{0}}^{2}-m_{\pi^{Q}}^{2}$ and the resulting fi t curves are shown as examples. From these fi ts we obtain

$$
\begin{array}{cll}
B_{0}=2.09(4), \Delta_{+}^{(0)}=0.0429(71), \Delta_{+}^{(m)}=2.28(31), \Delta_{0}^{(m)}=1.60(28), & \text { from } A_{0}, \\
B_{0}=2.06(4), \Delta_{+}^{(0)}=0.0466(51), \Delta_{+}^{(m)}=2.56(29), \Delta_{0}^{(m)}=1.94(27), & \text { from } P
\end{array}
$$

in lattice units. Since we have not measured masses of non-degenerate mesons, we simply apply eqs. (3.1) and (3.2) to estimate the kaon masses,

$$
\begin{array}{ll}
m_{K^{+}}^{2}=\alpha_{\mathrm{em}} \Delta_{+}^{(0)}+ & \left(B_{0}+\alpha_{\mathrm{em}} \Delta_{+}^{(m)}\right)\left(m_{f, s}+m_{\mathrm{res}, \mathrm{s}}+m_{f, u}+m_{\mathrm{res}, \mathrm{u}}\right), \\
m_{K^{0}}^{2}= & \left(B_{0}+\alpha_{\mathrm{em}} \Delta_{0}^{(m)}\right)\left(m_{f, s}+m_{\mathrm{res}, \mathrm{s}}+m_{f, d}+m_{\mathrm{res}, \mathrm{d}}\right) .
\end{array}
$$



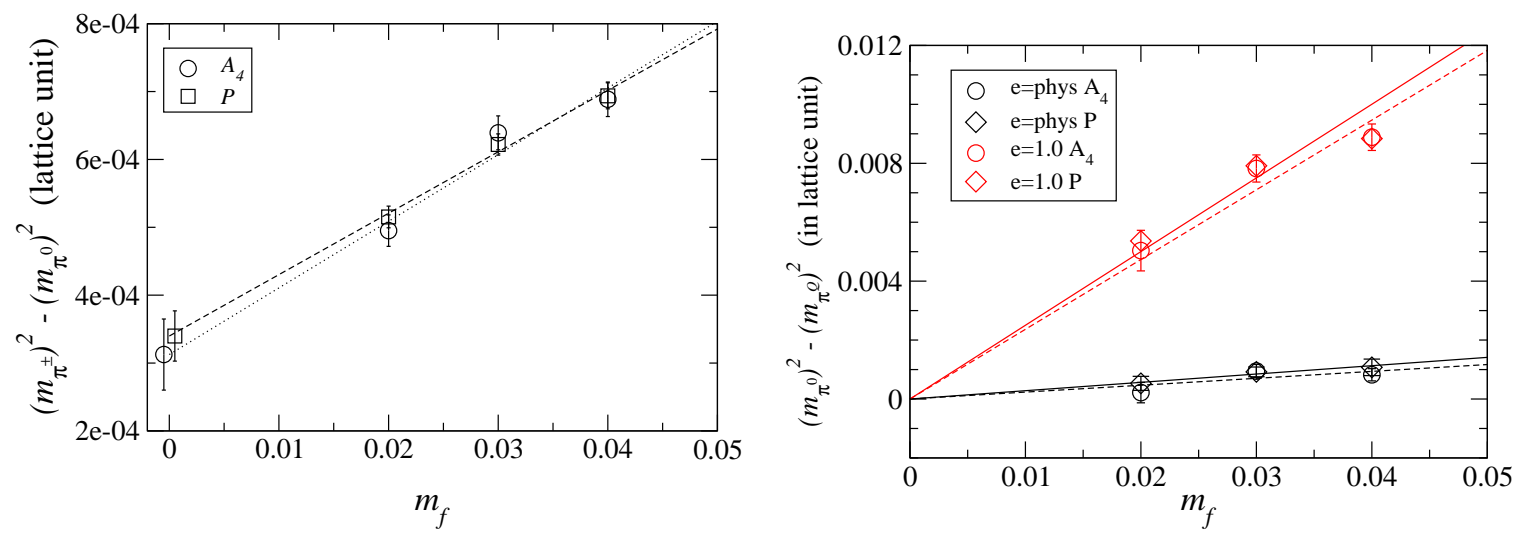

Figure 2: The $m_{f}$ dependence of $\pi^{+}-\pi^{0}$ splitting (left) and $\pi^{0}-\pi^{Q}$ splitting (right).

Using the lowest order ChPT expressions,

$$
\begin{aligned}
& m_{\pi^{0}}^{2}=2\left(B_{0}+\alpha_{\mathrm{em}} \Delta_{0}^{(m)}\right)\left(m_{f,+}+m_{\mathrm{res},+}\right) \\
& \left(m_{K^{+}}^{2}+m_{K^{0}}^{2}\right)=2 B_{0}\left(m_{f, s}+m_{f,+}+m_{\mathrm{res}, s}+m_{\mathrm{res},+}\right) \\
& +\alpha_{\mathrm{em}}\left(\Delta_{+}^{(0)}+\left(\Delta_{0}^{(m)}+\Delta_{+}^{(m)}\right)\left(m_{f, s}+m_{\mathrm{res}, s}\right)\right) \\
& \left(m_{K^{0}}^{2}-m_{K^{+}}^{2}\right)-\left(m_{\pi^{0}}^{2}-m_{\pi^{+}}^{2}\right)=2 B_{0}\left(m_{f,-}+m_{\mathrm{res},-}\right)+\alpha_{\mathrm{em}}\left(\Delta_{0}^{(m)}-\Delta_{+}^{(m)}\right)\left(m_{f, s}+m_{\mathrm{res}, s}\right),
\end{aligned}
$$

the results of eqs. (3.3) and (3.4), and the experimental values of the meson masses, we can determine $m_{f, \pm}$ and $m_{f, s}\left(O\left(\alpha_{\mathrm{em}} m_{f, \pm}\right)\right.$ terms have been neglected $)$. Using $a^{-1}=1.691 \mathrm{GeV}$, and the nonperturbative value of the quark mass renormalization factor $1 / Z_{m}=Z_{S}=0.62$ [8] $\left(m^{\overline{\mathrm{MS}}}(2 \mathrm{GeV})=\right.$ $Z_{m}\left(m_{f}+m_{\text {res }}\right)$ ), we obtain the following preliminary results for the quark masses (statistical error only)

$$
\begin{array}{ll}
m_{u}^{\overline{\mathrm{MS}}}(2 \mathrm{GeV}, \text { ref })=2.84(23) \mathrm{MeV}\left(A_{0}\right), & 2.89(8) \mathrm{MeV}(P), \\
m_{d}^{\overline{\mathrm{MS}}}(2 \mathrm{GeV}, \text { ref })=5.41(24) \mathrm{MeV}\left(A_{0}\right), & 5.49(13) \mathrm{MeV}(P), \\
m_{s}^{\overline{\mathrm{MS}}}(2 \mathrm{GeV}, \text { ref })=106.8(8) \mathrm{MeV}\left(A_{0}\right), & 106.5(5) \mathrm{MeV}(P) .
\end{array}
$$

While the statistical errors appear to be under control, we emphasize that our results are determined in the quenched approximation of QED, and we have used only $m_{f}=m_{\text {val }}=m_{\text {sea }}$ data points in our fi ts ('ref' in the above results is stressing it). It has been reported that in the pure QCD calculation partially-quenched (PQ) valence quark mass effects at the next-to-leading order in ChPT tend to increase the strange quark mass compared to the lowest-order extrapolation used here [5]. In addition, the naive defi nition of $m_{\text {res }}$ used in this analysis amounts to a constant shift downward of a few percent on each of the quark masses compared to the results in [5]. Thus our results should be considered preliminary. On the other hand, the effects of the EM interactions relative to pure QCD (and on the $m_{u}-m_{d}$ mass difference itself) should not depend signifi cantly on these details of the analysis, as long as both are carried out using the same method. We have determined $m_{+}^{\overline{\mathrm{MS}}}$ and $m_{s}^{\overline{\mathrm{MS}}}$ in pure QCD using the leading order method described here and fi nd that the EM interactions tend to decrease these masses by roughly one percent $\left(\sim O\left(\alpha_{\mathrm{em}}\right)\right)$ although it is statistically less signifi - 
cant. We are currently computing the EM splittings using PQ valence quarks and the conventional defi nition of $m_{\text {res }}$ to improve our determination of the quark masses.

\section{Discussion and prospects}

The systematic errors in this calculation are quickly surveyed below. In the measurements of neutral pions, we ignored the contribution from the disconnected diagram, which affects the determination of $\Delta_{0}^{(m)}$. While it is possible to calculate this diagram explicitly, the clean extraction of a signal is likely to be diffi cult.

In the study of the EM interactions, fi nite volume effects could be signifi cant as the photons are massless. We have estimated the fi nite volume effects by considering the vector-saturation model [9, 10, 11] and applying the physical volume of our lattices to this estimation. We find roughly a $+10 \%$ increase in $\Delta_{+}^{(0)}$. We expect a similar size of correction for the other new LECs, though we need to check this.

Although it was not discussed in detail here, we also need to take into account effects of the third dynamical quark and non-zero lattice spacing errors, as well as effects of the quenched QED approximation.

\section{References}

[1] For example, see the plenary review talks by T. Izubuchi and M. Lüscher in these proceedings.

[2] A. Duncan, E. Eichten and H. Thacker, Electromagnetic splittings and light quark masses in lattice QCD, Phys. Rev. Lett. 76, (1996) 3894, arXiv:hep-lat/9602005.

[3] D. B. Kaplan, A method for simulating chiral fermions on the lattice, Phys. Lett. B 288, (1992) 342, arXiv:hep-lat/9206013.

[4] Y. Shamir, Chiral fermions from lattice boundaries, Nucl. Phys. B 406, (1993) 90, arXiv:hep-lat/9303005.

[5] Y. Aoki et al. [RBC Collaboration], Lattice QCD with two dynamical flavors of domain wall fermions, arXiv:hep-lat/0411006.

[6] M. Hayakawa, T, Blum, T. Izubuchi and N. Yamada, Hadronic light-by-light scattering contribution to the muon g-2 from lattice QCD: Methodology, PoS(LAT2005) 353, arXiv:hep-lat/0509016.

[7] We thank H. Suganuma for a useful comment on this point.

[8] The RBC Collaboration, in progress; C. Dawson [RBC Collaboration], Dynamical domain wall fermions, Nucl. Phys. Proc. Suppl. 128, 54 (2004) [Nucl. Phys. Proc. Suppl. 129, 167 (2004)] [arXiv:hep-lat/0310055].

[9] T. Das, G. S. Guralnik, V. S. Mathur, F. E. Low and J. E. Young, Electromagnetic mass difference of pions, Phys. Rev. Lett. 18, (1967) 759.

[10] W. A. Bardeen, J. Bijnens and J. M. Gerard, Hadronic matrix elements and the $\pi^{+}-\pi^{0}$ mass difference, Phys. Rev. Lett. 62, (1989) 1343.

[11] G. Ecker, J. Gasser, A. Pich and E. de Rafael, The role of resonances in chiral perturbation theory, Nucl. Phys. B 321, (1989) 311. 